\title{
VALORACIÓN DE LA FUERZA MUSCULAR RESPIRATORIA EN PACIENTES CON ENFERMEDADES RESPIRATORIAS CRÓNICAS Y EN PERSONAS SALUDABLES
}

\author{
ASSESSMENT OF RESPIRATORY MUSCLE STRENGTH \\ IN PATIENTS WITH RESPIRATORY DISEASES CHRONIC \\ AND HEALTHY PEOPLE
}

\author{
SANTOS CHERO PISFIL \\ Universidad Norbert Wiener \\ Hospital Central Fuerza Aérea del Perú \\ RENZO DÍAZ RAMÍREZ \\ Hospital Central Fuerza Aérea del Perú \\ MARICIELO SÁNCHEZ QUIÑONES \\ Hospital Central Fuerza Aérea del Perú \\ YAJAIRA DÍAZ MAU \\ Hospital Central Fuerza Aérea del Perú \\ LUIS TITO GONZA \\ Hospital Guillermo Kaelin de la Fuente \\ LUIS CIEZA BOCANEGRA \\ Hospital Central Fuerza Aérea del Perú
}

\section{RESUMEN}

Los músculos respiratorios producen la expansión de la caja torácica, generando la ventilación y produciendo los ciclos ventilatorios. Los músculos de la respiración son el diafragma, los intercostales externos, los intercostales internos y la musculatura accesoria. El diafragma ejerce la fuerza necesaria para la expansión de la caja torácica en la fase inspiratoria ( $80 \%$ del trabajo aproximadamente). La pérdida de masa muscular se asocia a una disminución de la fuerza voluntaria-aproximadamente de 10 a $15 \%$ por cada década de vida-, que se evidencia a partir de los 50 ańos de edad. Entre los 70 y 80 años se produce un mayor declive, que alcanza el $30 \%$ de pérdida de masa muscular. El objetivo fue determinar la valoración de la fuerza muscular respiratoria en pacientes con enfermedades respiratorias crónicas y en personas saludables. La muestra estuvo conformada por 100 personas saludables ( 26 del sexo masculino y 74 del femenino) y 50 pacientes con enfermedades respiratorias crónicas estables, de los cuales 27 fueron masculinos y 23, femeninos (25 EPOC, 25 EPID), diagnosticados mediante espirometría, con edades comprendidas entre los 20 y 60 años, y mayores de 60 ańos, en dos grupos de estudio. Los criterios de inclusión en personas saludables fueron no deportistas, sin compromiso respiratorio no menor de 6 meses, que vivan en lima desde hace 10 ańos como mínimo; mientras que los pacientes respiratorios crónicos debían estar estables desde hace 3 meses como mínimo, con espirometría reciente, y que puedan comprender órdenes. Se excluyó a los que presentaban compromiso neurológico asociado y los que presentaban alteraciones cognitivas. La medición se realizó con un manovacuómetro portátil. La 
media de la fuerza muscular de la PImax y de la PEmax en personas saludables fue $81,83 \pm 24,39$ y $75,36 \pm 24,20$, respectivamente; y en las enfermedades respiratorias crónicas fue $56,40 \pm 28,35$ y $50,90 \pm 23,07$ ) con $\mathrm{p}=0,04$, respectivamente, teniendo en cuenta que los varones, en comparación con las mujeres, presentaron mayores valores tanto en saludables como en patológicos. Se concluye que los varones presentan mejores valores que las mujeres, tanto en los saludables como en los patológicos. Los pacientes más afectados son los de EPID; la edad es un factor influyente y la PEmax está más comprometida tanto por edad como por patología,

Palabras clave: presión inspiratoria máxima, presión espiratoria máxima, musculatura inspiratoria, enfermedad pulmonar obstructiva crónica, enfermedad pulmonar intersticial difusa.

\section{ABSTRACT}

The respiratory muscles produce expansion of the ribcage generating ventilation. As a result, it produces ventilator cycles. The respiratory muscles are: diaphragm, external intercostals, internal intercostals and accessory muscles. The diaphragm exerts the necessary force for expansion of the ribcage in the inspiratory phase (approximately $80 \%$ of the work). The loss of muscle mass is associated with a decrease in voluntary force, approximately $10-15 \%$ for each decade of life, the same that is evident from the 50-60 years old. Between 70 and 80 years old there is a greater decline that achieves the $30 \%$ of mass loss. The aim of this research was determine the valuation of the respiratory muscle strength in patients with chronic respiratory diseases and Healthy People. The sample consisted of 100 healthy people (26 male and 74 female) and 50 patients with stable chronic respiratory diseases, of this amount 27 were male and 23 were female (25 EPOC, 25 EPID), the sample was diagnosed by spirometry with ages between 20 to 60 years old and over 60 years. The criteria of inclusion in healthy people were: not athletes, who live in Lima since 10 years as a minimum and without respiratory compromise not less than 6 months. While the chronic respiratory patients must have been stable at least 3 months ago as minimum, with recent spirometry and can understand orders. We excluded patients that had associated neurological compromise also whom had cognitive impairment. The measurement was performed with a portable manovacuometer. The measure of muscle strength of the PImax and PEmax in healthy people were $(81,83$ $\pm 24,39$ y $75,36 \pm 24,20)$ And in diseases respiratory diseases were $(56,40 \pm 28,35$ y $50,90 \pm 23,07)$ with $\mathrm{p}=0,04$, respectively, we took into account that males with respect to females presented higher values in both healthy and pathological. In conclusions that males have better values in relation to both healthy and pathological women, the most affected disease is the EPID, the age is an influential factor and PEmax is more compromised by age and pathology.

Key words: maximum inspiratory pressure, maximum expiratory pressure, inspiratory musculature, chronic obstructive pulmonary disease, diffuse interstitial lung disease.

\section{INTRODUCCIÓN}

Los músculos respiratorios, encargados de la mecánica respiratoria, participan en la ventilación pulmonar y la expansión de la caja torácica. Entre los principales tenemos el diafragma, los intercostales externos e internos y la musculatura accesoria. El diafragma ejerce la fuerza necesaria para la expansión de la caja torácica 
en la fase inspiratoria (80\% del trabajo aproximadamente $)^{1}$. La pérdida de masa muscular se asocia a una disminución de la fuerza voluntaria, que se calcula entre 10 y $15 \%$ por cada década de vida, y se evidencia a partir de los 50-60 ańos de edad. A partir de los 70-80 años se produce un mayor declive, que alcanza el $30 \%$ de pérdida de masa ${ }^{2}$.

La pérdida de masa muscular es general y trae una serie de complicaciones. La medición de la fuerza de los músculos respiratorios es un factor importante que permite establecer el comportamiento respiratorio y el diagnóstico, así como cuantificar la gravedad de la disfunción y planificar el tratamiento a largo plazo.

La fuerza muscular respiratoria se mide mediante la manovacuometría, que valora la presión inspiratoria máxima (PImax) diafragmática y la presión espiratoria máxima (PEmax) de los músculos intercostales y abdominales.

La disminución de la fuerza muscular inspiratoria y su resistencia (reflejada en una menor PImax) propias del envejecimiento, llevan a una pérdida de la capacidad física, ocasionando ansiedad, depresión, aislamiento social y dependencia, llevando inclusive a un estado de invali$\mathrm{dez}^{3}$. Cabe considerar que, además de la edad, hay otros factores que influencian en la fuerza de los músculos respiratorios, como el retroceso elástico pulmonar, el crecimiento diferencial de las vías respiratorias, la distensibilidad de la pared torácica y las dimensiones del tórax ${ }^{4}$.

La medición de la manovacuometría, es aplicable en patologías respiratorias agudas y crónicas, alteraciones osteoarticulares y metabólicas, enfermedades neuromusculares, o en la evolución del ejercicio físico, mediante la terapéutica aplicada 5 .

La medición de la PImax y PEmax permite conocer y comparar los valo- res referenciales de peruanos saludables y aquellos con disfunción respiratoria crónica estable como los EPOC (Enfermedad Pulmonar Obstructiva Crónica) y EPID (Enfermedad Pulmonar Intersticial Difusa); actualmente solo hay referencias de mediciones internacionales, con diferente antropometría. El resultado de estas medidas nos servirá como referencia para prevenir recaídas por pérdida de fuerza muscular, entrenamiento en patologías respiratorias crónicas, así como la valoración del destete en pacientes sometidos a ventilación mecánica.

En las enfermedades respiratorias crónicas existen diversos grados de limitación ante el esfuerzo físico general y específico. Estas se correlacionan débilmente con variables de función pulmonar, por lo cual existen otros factores limitantes del esfuerzo físico entre ellos la disfunción de los músculos esqueléticos y los respiratorios que tienen importancia clínica; es de importancia la valoración de la debilidad de la fuerza diafragmática, determinada por las maniobras que se pueden realizar con la manovacuometría.

El objetivo de esta investigación fue determinar la fuerza muscular respiratoria en pacientes con enfermedades respiratorias crónicas y en personas saludables.

\section{MATERIALES Y MÉTODO}

La muestra estuvo conformada por 100 personas saludables (26 varones y 74 mujeres), trabajadores de un centro hospitalario, acompañantes de pacientes y estudiantes universitarios, y 50 pacientes con enfermedades respiratorias crónicas estables, 27 varones y 23 mujeres (25 EPOC, 25 EPID), diagnosticados mediante espirometría, con edades mayores a 20 años para ambos grupos. El estudio fue de tipo experimental, transversal, cuantitativo; los criterios de inclusión en 
personas saludables fueron no deportistas, sin compromiso respiratorio no menor de 6 meses, que vivan en lima desde hace 10 años como mínimo; los pacientes respiratorios crónicos debían estar estables por lo menos los 3 últimos meses, con espirometría reciente y que puedan comprender órdenes. Se excluyó a los que presentaban compromiso neurológico asociado y alteraciones cognitivas. La medición se realizó con un manovacuómetro portátil. El programa estadístico que se utilizó fue SPSS, versión 20 .

\section{RESULTADOS}

La media de la fuerza muscular de la PImax y PEmax en personas saludables fue $-81,83 \pm 24,39$ y $75,36 \pm 24,20$ ), res- pectivamente, mientras que en las enfermedades respiratorias crónicas fue $-56,40$ $\pm 28,35$ y $50,90 \pm 23,07)$ con $\mathrm{p}=0,04$, respectivamente, teniendo en cuenta que los varones en comparación con las mujeres presentaron mayores valores tanto en saludables como en patológicos (Tabla 1).

Respecto a las patologías, los que presentaron valores mayores fueron los EPOC, frente a los EPID (Tabla 2); se dividieron por grupos etarios: el grupo I estuvo constituido por pacientes de 20 a 29 años; el grupo II, de 30 a 39; el grupo III, de 40 a 49; el grupo IV de 50 a 59, y el grupo $\mathrm{V}$, mayores de 60 años. También se encontró que los valores fueron bastante parecidos en cuanto a la relación de grupos etarios y patologías (Tabla 4).

\section{TABLA 1}

\section{PImax-PEmax: pacientes respiratorios crónicos} y personas saludables según género

\begin{tabular}{ccccccccccc}
\hline Sexo & $\begin{array}{c}\text { Fuerza } \\
\text { muscular } \\
\text { respiratoria }\end{array}$ & $\begin{array}{c}\text { Pacientes con enfermedades } \\
\text { respiratorias crónicas }\end{array}$ & \multicolumn{5}{c}{ Personas saludables } \\
Frecuencia Media & $\begin{array}{c}\text { Desviación } \\
\text { estándar }\end{array}$ & $P^{*}$ & Frecuencia & Media & $\begin{array}{c}\text { Desviación } \\
\text { estándar }\end{array}$ & P* $^{*}$ \\
\hline Femenino & PIMAX & 23 & 49,13 & 24,98 & 0,09 & 74 & 77,88 & 22,62 & \\
& PEMAX & 23 & 45,13 & 19,86 & & 74 & 69,54 & 22,32 & 0,02 \\
Masculino & PIMAX & 27 & 62,59 & 30,01 & & 26 & 93,08 & 26,16 & \\
& PEMAX & 27 & 55,81 & 24,8 & 0,02 & 26 & 91,92 & 21,87 & 0,78 \\
\hline
\end{tabular}

* Prueba de t de Student

TABLA 2

Comportamiento de la PImax-PEmax según patologías

\begin{tabular}{lccc}
\hline Diagnóstico & PIMAX & PEMAX & P \\
\hline EPID & 54,28 & 50,4 & 0,122 \\
EPOC & 58,52 & 51,4 & 0,014 \\
\hline
\end{tabular}


TABLA 3A

Comportamiento de la PImax-PEmax en personas saludables según grupo etarios

\begin{tabular}{|c|c|c|c|c|c|c|c|}
\hline $\begin{array}{c}\text { Grupo } \\
\text { etario }\end{array}$ & Recuento & Media & $\begin{array}{l}\text { PIMAX } \\
\text { Desviación } \\
\text { estándar }\end{array}$ & $\mathrm{P}^{*}$ & Media & $\begin{array}{l}\text { PEMAX } \\
\text { Desviación } \\
\text { estándar }\end{array}$ & $\mathrm{P}^{*}$ \\
\hline Grupo I & 70 & 79,0 & 23,4 & \multirow{4}{*}{0,037} & 70,9 & 23,0 & \multirow{4}{*}{0,036} \\
\hline Grupo II & 22 & 87,1 & 26,8 & & 85,0 & 27,0 & \\
\hline Grupo III & 3 & 78,3 & 7,6 & & 73,3 & 7,6 & \\
\hline Grupo IV & 4 & 112,5 & 5,0 & & 100,0 & 8,2 & \\
\hline
\end{tabular}

TABLA 3B

Comportamiento de la media PImax-PEmax en enfermedades respiratorias crónicas según grupo etarios

\begin{tabular}{lccccccc}
\hline $\begin{array}{l}\text { Grupo } \\
\text { etario }\end{array}$ & Recuento & Media & $\begin{array}{c}\text { PIMAX } \\
\text { Desviación } \\
\text { estándar }\end{array}$ & $\mathbf{P}^{*}$ & Media & $\begin{array}{c}\text { PEMAX } \\
\text { Destándar }\end{array}$ & P \\
\hline Grupo III & 2 & 84 & 0 & & 88 & 0 & \\
Grupo IV & 2 & 110 & 0 & 0,005 & 100 & 0 & 0,000 \\
Grupo V & 46 & 52,87 & 26,48 & & 47,15 & & 19,92 \\
\hline
\end{tabular}

*ANOVA

TABLA 4

Comportamiento de la PImax-PEmax: relación grupos etarios-patología

\begin{tabular}{|c|c|c|c|}
\hline Diagnóstico & Grupo etario & PIMAX & PEMAX \\
\hline \multirow[t]{3}{*}{ EPID } & Grupo III & 84 & 88 \\
\hline & Grupo IV & 110 & 100 \\
\hline & Grupo V & 49,05 & 44,73 \\
\hline \multirow[t]{2}{*}{ EPOC } & Grupo IV & 110 & 100 \\
\hline & Grupo V & 56,38 & 49,38 \\
\hline
\end{tabular}

\section{DISCUSIÓN}

La finalidad del presente trabajo fue contrastar los parámetros encontrados en la literatura americana con los valores nacionales, obtenidos en este estudio, y, por tanto, debieran ser utilizados como referencia para nuestra población. Los pacientes estudiados presentaron una media de PImax de $-93,08 \pm 26,16$ y $-77,88 \pm 22,62)$, en varones y mujeres, respectivamente, (medida a partir del volumen residual). Según Black y Hya$\mathrm{tt}^{7}$, resultados de $75 \mathrm{cmH} 20$ en varones y $50 \mathrm{cmH} 2 \mathrm{O}$ para mujeres pueden ser considerados como "normales", y para la 
PEmax (medida a partir de la capacidad pulmonar total) un valor igual o mayor de $100 \mathrm{cmH} 2 \mathrm{O}$ para varones y de $80 \mathrm{~cm}$ $\mathrm{H} 2 \mathrm{O}$ para mujeres; en nuestros pacientes se registraron $91,92 \pm 21,87$ y 69,56 $\pm 22,32$. Sin embargo, Ordiales et al. ${ }^{8}$ encontraron en la PImax $-106 \pm 30,8$ y $-70,4 \pm 17,1$, mientras que en PEmax, $155,3 \pm 8$ y $91,4 \pm 16,8$, resultados distantes completamente a los encontrados en nuestro trabajo.

En un estudio realizado entre mujeres sedentarias y practicantes de hidrogimnasia $^{9}$ con una media de edad de 68,2 $\pm 5,8$ y 63,2 $\pm 2,5$, la PImax encontrada comparada fue de-51,5 $\pm 19,4$ y $-77,0 \pm 7,3$, respectivamente, con $\mathrm{p}=0.015$; aunque se evidencia una importante variabilidad en el primero de los grupos con una diferencia de $25,5 \mathrm{cmH} 2 \mathrm{O}$; no se encontró relación entre estos datos y el sobrepeso. Neder et al. ${ }^{10}$ presentan valores normales de referencia en mujeres de 60-69 años de la PImax, $-85,3 \pm 5,5$, resultados que se asemejan a los nuestros: $-77,88 \pm 22,62$, y revelan coincidencia con los valores que se muestran en la literatura.

La disminución de las PImax en los pacientes con EPOC es un hecho habitual y suficientemente documentado ${ }^{11}$; sin embargo, no existe unanimidad con respecto a los factores que contribuyen a esta disminución de la fuerza de los músculos inspiratorios; para la mayoría de autores es la hiperinsuflación el principal factor determinante, a través de la alteración que produce en la relación fuerza-longitud de la musculatura inspiratoria, particularmente el diafragma ${ }^{12}$. Pereira et al. ${ }^{13}$ comparan sanos y EPOC encontrando en la PImax $-90,8 \pm 12,7$ y $63,3 \pm 19,1$; respecto a los nuestros, presentaron $-81,83 \pm 24,39$ y $-58,52$, mientras que la PEmax fue $112,9 \pm 12,9$ y $109 \pm 17.6$. Los valores encontrados en nuestra muestra fueron $75,36 \pm 24,20$ y $51,4 \mathrm{p}=0,014$, los cuales divergen completamente. No es posible establecer cuáles son los motivos de las diferencias entre nuestros resultados y los de otras investigaciones sobre EPOC; aunque probablemente factores como el peso corporal, la existencia o no de acidosis y el grado de alteración gasométrica hayan influenciado ${ }^{14}$. En esa línea, es posible afirmar que uno de los factores que podrían influenciar en la debilidad muscular generalizada invocada por Rochester et al. ${ }^{12}$ es la hipercapnia, y en este sentido encuentran una correlación entre una PImax $<55$ cmH2O y los niveles de $\mathrm{PaCO} 2$; sin embargo, Pereira et al. ${ }^{13}$ encontraron que de los 25 pacientes solo 6 presentaron retención de $\mathrm{CO} 2$ con PImax< $55 \mathrm{~cm} \mathrm{H} 20$, mientras otros 6 tenían una PaCO2 >45 cmH20.

En relación a los cuadros respiratorios intersticiales como las EPID, no existen estudios de investigación, pero los resultados reflejan que su deterioro tiene mayor compromiso que los EPOC, ya que existen factores como la pérdida de elasticidad pulmonar que creemos influyen directamente sobre sus valores, y que la desventaja mecánica influenciada por la masa muscular se suma a los valores pobres encontrados en ambas mediciones de presión respiratoria máxima.

Llama la atención el grupo IV, tanto en saludables como en patológicos, ya que son los que obtienen los valores más altos en ambos tipos de presión máxima, para luego disminuir considerablemente en el grupo $\mathrm{V}$, como lo registran Pine et al. en su estudio desarrollado para examinar el deterioro funcional del sistema 
respiratorio relacionado con el envejecimiento, dividido en dos grupos: grupo A (50-59 años) y grupo B (60-69 años), además de examinar a varones $(n=14)$ y mujeres $(n=22)$. En sus resultados se observó deterioro significativo de la PI$\max (\mathrm{p}<0,05)$ y de la PEmax, entre los dos grupos conforme aumenta los años; mientras que los valores disminuidos del sexo femenino se debió a la disminución de la actividad física frente al envejecimiento; dichos resultados se asemejan a los obtenidos en nuestro estudio, lo que parece indicar que la práctica de la actividad física debe ser un factor influyente ${ }^{15}$.

\section{CONCLUSIONES}

La medición de la PImax y PEmax de los músculos respiratorios mediante manovacuometría es de gran utilidad, para su interpretación en la práctica de la fisiología respiratoria, para la evaluación y seguimiento de las diferentes patologías respiratoriad, inclusive en los pacientes que están en ventilación mecánica. Es sencilla, no invasiva, fácil de interpretar; sin embargo, depende del esfuerzo de cada persona, aun ejecutándola de forma estandarizada con el control adecuado, ya que se evidencia que tanto en saludables como patológicos, la edad es un factor influyente.

Se concluye que: 1) los varones presentan mejores valores respecto a las mujeres, tanto en los saludables como en patológicos; 2) los pacientes más afectados son los de EPID; 3) en la edad de 50-59 (grupo IV), alcanzan sus máximos valores en las personas saludables y patológicos, 4) la PEmax está más comprometida tanto por edad como por patología; 5) los valores referenciales de los saludables, según las diferentes ecuaciones presentes en la literatura, no concuerdan con los valores obtenidos ${ }^{16}$; 6) se debe continuar la investigación con una muestra mayor en ambos grupos de estudio.

\section{REFERENCIAS BIBLIOGRÁFICAS}

1. Mañanas Villanueva M. Análisis de la actividad muscular respiratoria mediante técnicas temporales, frecuenciales y estadísticas. Universidad Politécnica de Catalunya, 1999.

2. Izquierdo Rendín M. Biomecánica y bases neuromusculares de la actividad física y del deporte. Edit Panamericana, 2008.

3. González-Montesinos J et al. Efectos del entrenamiento de la musculatura respiratoria sobre el rendimiento.RevAndalMed Deporte 2012;5:163-70.

4. Johan A, Chan CC, Chia HP, Chan OY, Wang Y'T. Maximal respiratory pressures in adult Chinese, Malays and Indians. EurRespir J 1997; 10(12):2825-2828.

5. Domínguez Flores $M$ et al. Guía para el diagnóstico y tratamiento de las alteraciones respiratorias en las enfermedades neuromusculares. RevNeumolCirTorax, vol.70- num, 1:5-70, 2011,

6. Sánchez De León R, Bases de la neumología clínica. Universidad central de Venezuela, 2004.

7. Black LF, Hyatt RE. Maximal respiratory pressures: normal values and relationship to age and sex. Am RevRespirDis 1969;99(5):696-702.

8. Ordiales F et al. Presiones respiratorias estáticas máximas. Importancia del estudio de los valores de referencia normales. Arch de Bronconeumología 1995: 507-511. 
9. Ali Carder $\mathrm{S}$ et al. Comparación de la fuerza de la musculatura inspiratoria entre mujeres mayores sedentarias y practicantes de hidrogimnasia. RevEspGeriatrGerontol. 2007;42(5):271-5.

10. Neder JA, Andreoni S, Lerario MC, Nery LE. Reference values for lung function tests. II. Maximal respiratory pressures and voluntary ventilation. Braz J Med Biol Res. 1999;32(6):719-27.

11. Rochester DR, Braun NMT., Arora NS.: Respiratory muscle strength in chronic obstructive pulmonary disease. Am Rev. Respir. Dis. 1979; 119: 151-154.

12. Rochester DR, Braun NMT.: Determinants of maximal inspiratory pressure in chronic obstructive pulmonary disease. Am Rev. Respir. Dis. 1985; 132: 42-47.
13. Pereira Vega A et al. Evaluacion de la fuerza de los músculos respiratorios en la enfermedad pulmonar obstructiva crónica (EPOC). Neumosur, revista de la Asociación de neumólogos del sur vol.2, número 2, diciembre, 1990.

14. Pangelly LD., Alderson AM., Milic-Emili J.: Mechanics of the diaphragm. J. Appl. Physiol 1971; 30: 797-805 .

15. Pine MJ. The effects of ageing on respiratory muscle function and performance in older adults. Journal Science Medical Sport. 2007;10(1):36-44.

16. Wilson SH, Cooke NT, Edwards RH, Spiro SG. Predicted normal values for maximal respiratory pressure in Caucasian adults and children. Tho$\operatorname{rax} 1984 ; 39(7)$ : 535-538. 